\title{
The Role of Candida Albicans and Streptococcus Mutans Spent Culture Supernatant in Single and Dual-Species Biofilm
}

\author{
Regis WFM, Reis ACM, Rocha FR, Guedes SFF, Maia DCBS and Rodrigues LKA* \\ Department of Operative Dentistry, Brazil
}

*Corresponding author: Rodrigues LKA, Faculty of Pharmacy, Dentistry and Nursing, Department of Operative Dentistry, Brazil

\begin{tabular}{|c|c|}
\hline ARTICLE INFO & ABSTRACT \\
\hline 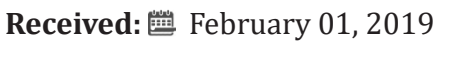 & Citation: Regis WFM, Reis ACM, Rocha FR, Guedes SFF, Maia DCBS, Rodrigues LK \\
\hline Published: 慧 February 15, 2019 & $\begin{array}{l}\text { Role of Candida Albicans and Streptococcus Mutans Spent Culture Supernatant in Single } \\
\text { and Dual-Species Biofilm. Biomed J Sci \& Tech Res 14(4)-2019. BJSTR. MS.ID.002588. }\end{array}$ \\
\hline
\end{tabular}

\section{Short Communication}

The association of bacteria with fungal species in biofilms can provide substrates, metabolites and growth factors in certain circumstances [1], and these interactions or "communication" mechanisms between distinct species living in biofilms, which occur when microorganisms exchange chemical signals, are known as quorum sensing (QS) [2]. Microorganisms within biofilms can also belong to the same species, and the generated signals can freely spread and diffuse across the cell membrane into the medium, thereby orchestrating biofilm formation [3]. The QS molecules which have been found in the spent culture supernatant (SCS) of bacterial and fungal cultures have been noted as regulators of virulence mechanisms related to biofilm formation, especially in pathogenic biofilms [4]. Frequently, S. mutans and C. albicans are found together in oral biofilms [1]. Thus, considering the polymicrobial characteristic of dental caries, investigating the way that interactions between microbes affect biofilm formation and cell morphology is essential to understanding the pathogenesis of this disease [5]. Therefore, this study aimed to evaluate the influence of $C$. albicans and S. mutans SCS, combined or not, in the biofilm formation of these microorganisms grown in their singlespecies and dual-species biofilms.

\section{Subjects and Methods}

Strains of C. albicans (ATCC 10231) and S. mutans (UA 159) were grown in YNB and BHI, respectively, for $18 \mathrm{~h}$, at $37^{\circ} \mathrm{C}$ under microaerophilic conditions [6]. The microbial cultures were

adjusted to a $0.5 \mathrm{Mc}$ Farland standard (equivalent to $1.5 \times 10^{8}$ $\mathrm{CFU} / \mathrm{mL}$ ) in YNB broth supplemented with $1 \%$ glucose $(\mathrm{w} / \mathrm{v})$ and BHI broth supplemented with $1 \%$ sucrose $(\mathrm{w} / \mathrm{v})$, respectively. The microorganisms were inoculated separately into the wells of in microtiter plate and incubated at $37{ }^{\circ} \mathrm{C}\left(5 \% \mathrm{CO}_{2}\right)$ for $48 \mathrm{~h}$, with culture medium replaced every day. After this period, for SCS obtainment, the biofilms were centrifuged at 1,300 rpm (NT-835, Novatecnica, Piracicaba, SP, Brazil) and SCS filtered with $0.22-\mu \mathrm{m}$ membranes filters as previously described $[7,8]$. The single and dual- species biofilms were grown in vitro in the presence of the studied SCS combined or isolated, cultured in RPMI-1640 medium for $48 \mathrm{~h}$ [1]. Following this period, the biofilms were collected, the biomass was determined [9] and soluble and insoluble extracellular polysaccharides were assessed [10]. The polysaccharide analysis was expressed as total extracellular polysaccharides (tEPS) by adding the values of soluble plus insoluble extracellular polysaccharides. Data were expressed as a mean and standard deviation, and group comparison was performed by Student T-test or ANOVA followed by Bonferroni's post-hoc test. Graph Pad Prism version 5.0 was used for data analysis and a $95 \%$ confidence value was considered.

\section{Results and Discussion}

Our results showed no difference in $S$. mutans biomass when in contact with both SCS tested (Figure 1a). However, there was an increase in $C$. albicans biomass in the presence of its own SCS 
(Figure 1b). In the dual-species biofilms, the $S$. mutans biomass was statistically significant $(\mathrm{p}<0.001)$, decreased if co-cultured with the $C$. albicans SCS compared with the control group (Figure 1c), while the dual-species biofilm showed no difference of $C$. albicans counts if compared with the control group (Figure 1d). Regarding the total extracellularpolysaccharides (tEPS) produced by $S$. mutans when in contact with its own SCS and C. albicans SCS ( $\mathrm{p}=0.0002)$ (Figure 2a), a significant increase in their amounts was observed $(\mathrm{p}<0.0001)$. Additionally, in a single-species biofilm of $C$. albicans a markedly increase in tEPS production occurred when the biofilm was cultured in contact with $S$. mutans SCS ( $p=0.0025)$ (Figure $2 \mathrm{~b}$ ) as well as in the tEPS production in the dual-species biofilms cultured $S$. mutans SCS and when cultured in the presence of both SCS ( $\mathrm{p}<0.0001$ ) (Figure 2C). In this study, we investigated the role of $S$. mutans and C. albicans SCS on the formation of single- and dualspecies biofilms. We chose to study these microorganisms because previous investigations have shown that the interaction between them can modulate the development of dental caries $[7,8]$.

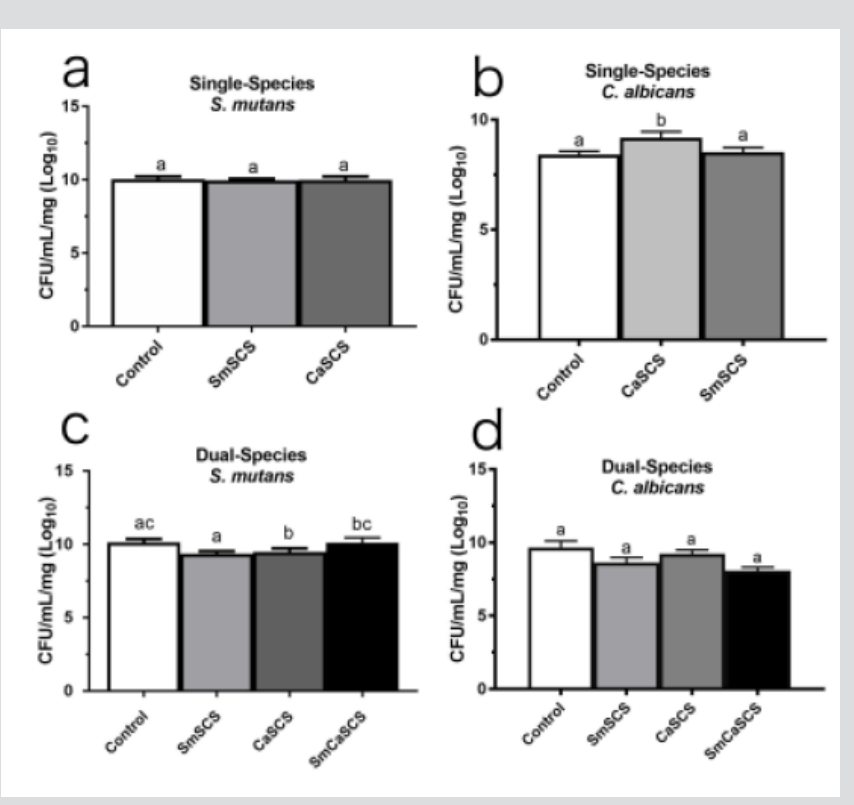

Figure 1: Mean and standard deviation of the CFU/mL/mg (Log10) obtained of single-species (a and b) and dual-species (c and d) biofilms formed by S. mutans (Sm) and C. albicans (Ca) grown without Spent Culture Supernatant (SCS) (control), in contact with S. mutans SCS (SmSCS), with C. albicans SCS (CaSCS), and with S. mutans plus C. albicans SCS (SmCaSCS).

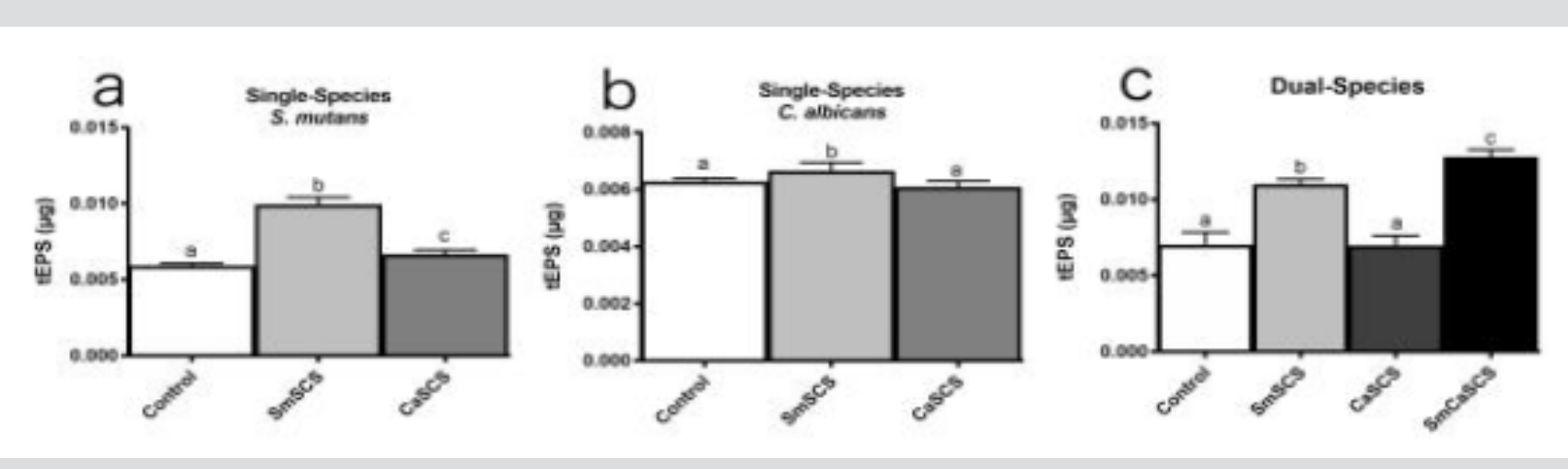

Figure 2: Mean and standard deviation of the values of total polysaccharides of (tEPS) single- and dual-species biofilms of $S$. mutans and C. albicans produced by S. mutans (Sm) and C. albicans (Ca) without Spent Culture Supernatant (SCS) (control), in contact with S. mutans SCS (SmSCS), with C. albicans SCS (CaSCS) and with S. mutans plus C. albicans SCS (SmCaSCS).

According to our findings, both SCS did not affect the biomass of $S$. mutans single-species biofilms $(\mathrm{CFU} / \mathrm{mL} / \mathrm{mg})$. However, a significant increase in tEPS $(\mathrm{p}<0.0001)$ was observed. This result can be explained by the fact that the alterations caused in the biofilms by the SCS might not be related to growth kinetics, but to polysaccharides production, which corroborates with previous results $[3,11]$. Extracellular products of $C$. albicans affect the activation of genes related to polysaccharides production in $S$. 
mutans [12]. In C. albicans single-species biofilms, an increase in the biomass and insoluble polysaccharide production when cultured in the presence of S. mutans SCS [11] was found. In this study, S. mutans SCS favored C. albicans biofilm formation and polysaccharides production. Our results agree with previous studies which have shown a symbiotic relationship between $C$. albicans and S. mutans in dental caries biofilms $[3,7,11]$ and indicate that $C$. albicans cultured with $S$. mutans increases biofilm biomass. Moreover, extracellular molecules produced by $S$. mutans enhances $C$. albicans filamentation. Our results disagree with studies reporting that $S$. mutans quorum sensing molecules negatively interfere with $C$. albicans biofilm formation and that the quorum sensing competence inducing peptide (CSP) molecules act by decreasing $C$. albicans viability in co-cultures $[3,13]$.

We can suppose that only a physiological concentration of CSP produced by $S$. mutans was secreted and higher amounts would be necessary to affect $C$. albicans. Although $C$. albicans SCS could positively influence $S$. mutans cells, interactions between pathogenic bacteria and fungi remain unknown. This result confirms the symbiotic relationship between these microorganisms also previously described $[7,11]$. This study provides new information on interactions between microorganisms of different kingdoms In summary, the $S$. mutans SCS increased the $C$. albicans biomass in single-species biofilms and the tEPS production of $S$. mutans and C. albicans in both models of biofilms. In contrast, C. albicans SCS reduced the biomass of $S$. mutans in single-species biofilms. Other studies must be performed to clarify the molecular mechanisms involved in the pathogenesis of dental caries biofilms formed by the microorganisms studied in this investigation.

\section{Acknowledgements}

The first author was supported by a CAPES (National Council for the Improvement of Higher Education) scholarship funded by the Brazilian government during her Master degree course, when this research was conducted.

\section{ISSN: 2574-1241}

DOI: 10.26717.BJSTR.2019.14.002588

Rodrigues LKA. Biomed J Sci \& Tech Res

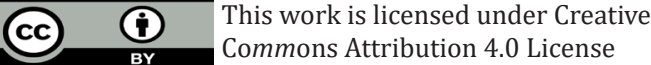

Submission Link: https://biomedres.us/submit-manuscript.php

\section{References}

1. Arzmi MH, Dashper S, Catmull D, Cirillo N, Reynolds EC, et al. (2015) Coaggregation of Candida albicans, Actinomyces naeslundii and Streptococcus mutans is Candida albicans strain dependent. FEMS Yeast Res 15(5): 1-7.

2. Fernandes RA, Monteiro DR, Arias LS, Fernandes GL, Delbem ACB, et al. (2016) Biofilm formation by C. albicans and Streptococcus mutans in the presence of farnesol: a quantitative evaluation. Biofouling 32(3): 329338.

3. Falsetta ML, Klein MI, Colonne PM, Scott-Anne K, Gregoire S, Pai CH (2014) Symbiotic relationship between Streptococcus mutans and $C$. albicans synergizes virulence of plaque biofilms in vivo. Infect Immun 82(5): 1968-1981.

4. Li YH, Tian X (2012) Quorum sensing and bacterial social interactions in biofilms. Sensors 12(3): 2519-2538.

5. Santos AD, Sa EACD, Gaziri LCJ, Felipe I (2002) Treatment of serum with supernatants from cultures of $C$. albicans reduces its serum-dependent phagocytosis. Braz J Microbiol 33(1): 79-83.

6. Arias LS, Delbem ACB, Fernandes RA, Barbosa DB, Monteiro DR (2016). Activity of tyrosol against single and mixed-species oral biofilms. J. Appl Microbiol 120(5): 1240-1249.

7. Barbosa JO, Rossoni RD, Vilela SFG, DE Alvarenga JA, Velloso M, et al. (2016) Streptococcus mutans can modulate biofilm formation and attenuate the virulence of $C$. albicans. PLoS One 11(3): 16.

8. Lins de Sousa D, Araújo Lima R, Zanin IC, Klein MI, Janal MN, et al (2015) Effect of twice-daily blue light treatment on matrix- rich biofilm development. PloS one 10(7): e0131941.

9. Dubois M, Gilles K, Hamilton J, Rebers PA, Smith F (1956) A colorimetric method for the determination of sugars. Nature 168: 350- 356.

10. Sztajer H, Szafranski SP, Tomasch J, Reck M, Nimtz M, et al. (2014) Crossfeeding and interkingdom communication in dual-species biofilms of Streptococcus mutans and C. albicans. ISME J 11(8): 2256

11. Feldman M, Ginsburg I, Al-Quntar A, Steinberg D (2016) Thiazolidinedione-8 alters symbiotic relationship in C. albicans-S. mutans dual species biofilm. Front Microbiol 7: 12 .

12. Jarosz LM, Deng DM, Van Der, Mei HC, Crielard W, Krom BP (2009) Streptococcus mutans competence-stimulating peptide inhibits $C$. albicans hypha formation. Eukaryot Cell 8(11): 1658-1664

13. Koo H, Bowen WH (2014) Candida albicans and Streptococcus mutans: a potential synergistic alliance to cause virulent tooth decay in children. Future Microbiol 9(2) :12.

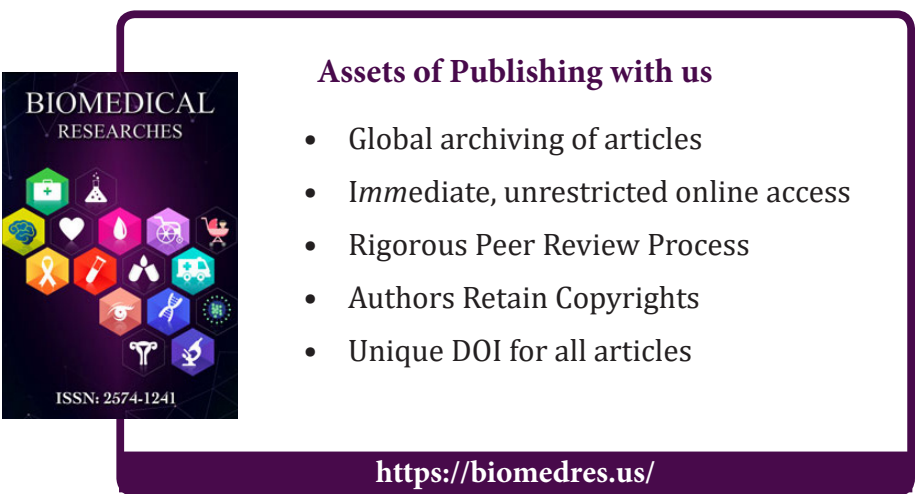

Copyright@ Rodrigues LKA| Biomed J Sci \& Tech Res| BJSTR. MS.ID.002588. 